\title{
Common fixed points of monotone Lipschitzian semigroups in Banach spaces
}

\author{
M. Bachar ${ }^{\mathrm{a}}$, Mohamed A. Khamsi ${ }^{\mathrm{b}, \mathrm{c}, *}$, W. M. Kozlowski ${ }^{\mathrm{d}}$, M. Bounkhel ${ }^{\mathrm{a}}$ \\ ${ }^{a}$ Department of Mathematics, College of Science, King Saud University, Saudi Arabia. \\ ${ }^{b}$ Department of Mathematical Sciences, The University of Texas at El Paso, El Paso, TX 79968, U.S.A. \\ ${ }^{c}$ Department of Mathematics \& Statistics, King Fahd University of Petroleum and Minerals, Dhahran 31261, Saudi Arabia. \\ ${ }^{d}$ School of Mathematics and Statistics, University of New South Wales, Australia.
}

Communicated by F. Vetro

\begin{abstract}
In this paper, we investigate the existence of common fixed points of monotone Lipschitzian semigroup in Banach spaces under the natural condition that the images under the action of the semigroup at certain point are comparable to the point. In particular, we prove that if one map in the semigroup is a monotone contraction mapping, then such common fixed point exists. In the case of monotone nonexpansive semigroup we prove the existence of common fixed points if the Banach space is uniformly convex in every direction. This assumption is weaker than uniform convexity.
\end{abstract}

Keywords: Common fixed point, fixed point, monotone contraction mappings, monotone nonexpansive mappings, monotone Lipschitzian semigroup.

2010 MSC: 47H10, 47H09.

(C)2018 All rights reserved.

\section{Introduction}

Existence of fixed points for contractions and nonexpansive mappings acting in Banach spaces, as well as of common fixed points for semigroups of such mappings, has been intensely studied for more than half a century. Following the publication of the work of Ran and Reurings [18], a new research direction was developed where the Banach space is endowed with a partial order and the Lipschitzian assumption is only made for some comparable (in the order sense) elements. Such assumptions are generally much weaker and they make sense from the applications point of view. For example, in financial mathematics, incomparable elements may be interpreted as belonging to different markets and hence a Lipschitz type condition for such incomparable elements is meaningless. For more information on the recent status of

\footnotetext{
*Corresponding author

Email addresses: mbachar@ksu.edu.sa (M. Bachar), mohamed@utep.edu (Mohamed A. Khamsi), w.m.kozlowski@unsw.edu.au (W. M. Kozlowski), bounkhel@ksu.edu.sa (M. Bounkhel)
}

doi: $10.22436 /$ jnsa.011.01.06

Received: 2017-10-14 Revised: 2017-11-17 Accepted: 2017-11-20 
the monotone fixed point theory the reader is referred to the survey article by Bachar and Khamsi [3], see also [1].

The theory of common fixed points for semigroups of monotone contractions and monotone nonexpansive mappings is still in its infancy. The theory is interesting both from the purely theoretical point of view and because of nontrivial applications to various domains including differential and integral equations, and dynamical systems, see [2, 13, 16, 17]. In [2] Bachar and Khamsi proved some results on approximate fixed point sequences for such semigroups, while Kozlowski [13] demonstrated existence of common fixed points for monotone contractive and monotone nonexpansive semigroups. The current paper generalizes the existence results of [13].

Since this work deals with the metric fixed point theory, we recommend the books $[9,11]$.

\section{Preliminaries}

Throughout this work, $(X,\|\cdot\|)$ is a Banach vector space partially ordered by $\preceq$. We will assume that order intervals are closed and convex. Recall that an order interval is any of the subsets

$$
[a, \rightarrow)=\{x \in X ; a \preceq x\},(\leftarrow, b]=\{x \in X ; x \preceq b\}, \text { or }[a, b]=[a, \rightarrow) \cap(\leftarrow, b]
$$

for any $a, b \in X$. We will say that two elements $x, y \in X$ are comparable if $x \preceq y$ or $y \preceq x$.

Next we give the definition of monotone Lipschitzian mappings.

Definition 2.1. Let $C$ be a nonempty subset of $X$. A map $T: C \rightarrow X$ is said to be

(a) monotone if $T(x) \preceq T(y)$ whenever $x \preceq y$;

(b) monotone Lipschitzian if $\mathrm{T}$ is monotone and there exists $K \geqslant 0$ such that

$$
\|T(x)-T(y)\| \leqslant K\|x-y\|
$$

for any comparable elements $x, y \in C$.

If $K<1$ (resp. $K=1$ ), then $T$ is said to be a monotone contraction (resp. monotone nonexpansive mapping). A point $x$ is a fixed point of $T$ if $T(x)=x$. The set of all fixed points of $T$ will be denoted by $\operatorname{Fix}(\mathrm{T})$.

Remark 2.2. Note that if $\mathrm{T}: \mathrm{C} \rightarrow \mathrm{C}$ is a monotone contraction mapping, then any two comparable fixed points of $\mathrm{T}$ are equal. Indeed, let $z_{1}$ and $z_{2}$ be two fixed points of $\mathrm{T}$ which are comparable. Since $\mathrm{T}$ is a monotone contraction mapping, there exists $\mathrm{K}<1$ such that

$$
\left\|z_{1}-z_{2}\right\|=\left\|\mathrm{T}\left(z_{1}\right)-\mathrm{T}\left(z_{2}\right)\right\| \leqslant \mathrm{K}\left\|z_{1}-z_{2}\right\| .
$$

This will clearly force $z_{1}=z_{2}$.

Definition 2.1 is extended to the case of a one parameter semigroup of mappings.

Definition 2.3. Let $C$ be a nonempty subset of $X$. A one-parameter family $\mathcal{F}=\{T(t) ; t \geqslant 0\}$ of mappings from $C$ into $C$ is said to be a monotone semigroup if $\mathcal{F}$ satisfies the following conditions:

(i) $\mathrm{T}(0) x=x$ for $x \in C$;

(ii) $\mathrm{T}(\mathrm{t}+\mathrm{s})=\mathrm{T}(\mathrm{t}) \circ \mathrm{T}(\mathrm{s})$ for $\mathrm{t}, \mathrm{s} \in[0, \infty)$;

(iii) for each $t \geqslant 0, T(t)$ is a monotone mapping.

Definition 2.4. A monotone semigroup $\mathcal{F}$ is called a monotone Lipschitzian semigroup if there exists $K \geqslant 0$ such that

$$
\|T(t) x-T(t) y\| \leqslant K\|x-y\|
$$

for any comparable elements $x, y \in C$ and any $t \geqslant 0$. If $K<1$, then $\mathcal{F}$ will be called a monotone contractive semigroup . Similarly, if $K=1$, then $\mathcal{F}$ will be called a monotone nonexpansive semigroup.

The set of all common fixed points of $\mathcal{F}$ is defined by $\operatorname{Fix}(\mathcal{F})=\bigcap_{t \geqslant 0} \operatorname{Fix}(T(t))$. 


\section{The monotone contraction case}

Our first result generalizes Theorem 3.1 [13], where the author assumed $C$ to be weakly compact.

Theorem 3.1. Let $\mathrm{C}$ be a closed convex nonempty subset of $\mathrm{X}$. Let $\mathcal{F}=\{\mathbf{T}(\mathrm{t})\}_{\mathrm{t} \geqslant 0}$ be a monotone semigroup defined on $\mathrm{C}$. Assume that there exists $\mathrm{t}_{0}>0$ such that $\mathrm{T}\left(\mathrm{t}_{0}\right)$ is a monotone contraction mapping. Moreover, assume there exists $x_{0} \in C$ such that $x_{0} \preceq T(t) x_{0}$ (resp. $T(t) x_{0} \preceq x_{0}$ ), for any $t \geqslant 0$. Then there exists a common fixed point $z \in \operatorname{Fix}(\mathcal{F})$ such that $x_{0} \preceq z$ (resp. $z \preceq x_{0}$ ). Moreover, if $\mathrm{C}$ is bounded, then we have $\lim _{\mathrm{t} \rightarrow \infty}\left\|\mathrm{T}(\mathrm{t}) \mathrm{x}_{0}-z\right\|=0$.

Proof. Without loss of generality, we assume $x_{0} \preceq T(t) x_{0}$ for any $t \geqslant 0$. Since $T\left(t_{0}\right)$ is a monotone contraction, then $\left\{T^{n}\left(t_{0}\right) x_{0}\right\}$ is a Cauchy sequence. Since $C$ is a closed subset of a Banach space, there exists $z \in \mathrm{C}$ such that $\lim _{n \rightarrow \infty} \mathrm{T}^{\mathrm{n}}\left(\mathrm{t}_{0}\right) \mathrm{x}_{0}=z$. Since order intervals are closed, then $z$ is a fixed point of $\mathrm{T}\left(\mathrm{t}_{0}\right)$. Indeed, the sequence $\left\{T^{n}\left(t_{0}\right) x_{0}\right\}$ is monotone increasing. Hence we have $T^{m}\left(t_{0}\right) x_{0} \in\left[T^{n}\left(t_{0}\right) x_{0}, \rightarrow\right)$ for any $n \in \mathbb{N}$ and $m \geqslant n$. Since order intervals are closed, we conclude that $z \in\left[T^{n}\left(t_{0}\right) x_{0}, \rightarrow\right)$, i.e., $T^{n}\left(t_{0}\right) x_{0} \preceq z$ for any $n \in \mathbb{N}$. Since $T\left(t_{0}\right)$ is a monotone contraction, there exists $K<1$ such that $\left\|T\left(t_{0}\right) x-T\left(t_{0}\right) y\right\| \leqslant K\|x-y\|$ for any comparable elements $x, y \in C$. In particular, we have

$$
\left\|T^{n+1}\left(t_{0}\right) x_{0}-T\left(t_{0}\right) z\right\| \leqslant K\left\|T^{n}\left(t_{0}\right) x_{0}-z\right\|
$$

for any $n \in \mathbb{N}$. Clearly, this will force $T\left(t_{0}\right) z=z$ as claimed. Let us prove that $z$ is a common fixed point of $\mathcal{F}$. Note that we have

$$
T^{n}\left(t_{0}\right) \circ T(t)=T\left(n t_{0}+t\right)=T(t) \circ T^{n}\left(t_{0}\right)
$$

for any $n \in \mathbb{N}$ and $t \geqslant 0$. Hence

$$
T^{n}\left(t_{0}\right)(T(t) z)=T(t)\left(T^{n}\left(t_{0}\right) z\right)=T(t) z,
$$

i.e., $T(t) z$ is also a fixed point of $T\left(t_{0}\right)$. Next, we prove that $z \preceq T(t) z$. Indeed, we have

$$
\mathrm{T}^{\mathrm{n}}\left(\mathrm{t}_{0}\right) \mathrm{x}_{0} \preceq \mathrm{T}^{\mathrm{n}}\left(\mathrm{t}_{0}\right)\left(\mathrm{T}(\mathrm{t}) \mathrm{x}_{0}\right)=\mathrm{T}(\mathrm{t})\left(\mathrm{T}^{\mathrm{n}}\left(\mathrm{t}_{0}\right) \mathrm{x}_{0}\right) \preceq \mathrm{T}(\mathrm{t}) z
$$

for any $n \in \mathbb{N}$. Since $(\leftarrow, T(t) z]$ is closed and $\left\{T^{n}\left(t_{0}\right) x_{0}\right\}$ converges to $z$, we conclude that $z \preceq T(t) z$ as claimed. Using Remark 2.2, we conclude that $T(t) z=z$ as claimed. Finally, assume $C$ is bounded. Set $\delta(C)=\operatorname{diam}(C)$. Since $t_{0}>0$, then for any $t \geqslant 0$, there exists a unique $n \in \mathbb{N}$ such that $t=n t_{0}+\varepsilon$, with $0 \leqslant \varepsilon<\mathrm{t}_{0}$. Hence

$$
\mathrm{T}(\mathrm{t}) \mathrm{x}_{0}-z=\mathrm{T}(\mathrm{t}) \mathrm{x}_{0}-\mathrm{T}(\mathrm{t}) z=\mathrm{T}\left(\mathrm{nt}_{0}\right)\left(\mathrm{T}(\varepsilon) \mathrm{x}_{0}\right)-\mathrm{T}\left(\mathrm{nt} \mathrm{t}_{0}\right)(\mathrm{T}(\varepsilon) z),
$$

which implies $\left\|T(t) x_{0}-z\right\| \leqslant K^{n}\left\|T(\varepsilon) x_{0}-T(\varepsilon) z\right\|$, since $T(\varepsilon)$ is monotone and $x_{0} \preceq z$. Therefore, we have $\left\|T(t) x_{0}-z\right\| \leqslant K^{n} \delta(C)$. Note that $n \rightarrow \infty$ if and only if $t \rightarrow \infty$, which implies $\lim _{t \rightarrow \infty}\left\|T(t) x_{0}-z\right\|=0$.

In the next section, we will discuss the case of monotone nonexpansive semigroups. Note that the nonexpansive condition complicates the existence of a fixed point drastically and necessitates geometric conditions satisfied by the underlined space $[5-7,10,12,14,15,19,20]$.

\section{Monotone nonexpansive semigroups in Banach spaces}

The main tool used to prove the existence of a common fixed point for a monotone nonexpansive semigroup is the existence and uniqueness of a minimum point of a real-valued convex function. In order to obtain such minimum point, we will need the Banach space to have a nice geometric property.

Definition $4.1([8,21])$. Let $(X,\|\cdot\|)$ be a Banach space. Fix $\varepsilon \in(0,2]$ and $z \in X$ such that $\|z\|=1$. Consider the set

$$
D_{z, \varepsilon}=\{(x, y) \in X \times X ;\|x\| \leqslant 1,\|y\| \leqslant 1, x-y= \pm\|x-y\| z, \text { and }\|x-y\| \geqslant \varepsilon\} .
$$

We say that a Banach space $(X,\|\|$.$) is uniformly convex in the direction z \in X$ if $\delta(\varepsilon, z)>0$, where

$$
\delta(\varepsilon, z)=\inf \left\{1-\left\|\frac{x+y}{2}\right\| ;(x, y) \in D_{z, \varepsilon}\right\} .
$$


This property was initiated by Garkavi [8] while studying the concept of Chebyshev centers. In the work of Zizler [21], it is shown that any separable Banach space has an equivalent norm which is uniformly convex in every direction. This result is crucial since uniformly convex Banach spaces are super-reflexive [4]. Hence the class of uniformly convex Banach spaces is a lot smaller that the class of Banach spaces which are uniformly convex in every direction. Moreover, it is easy to check that if $(X,\|\cdot\|)$ is uniformly convex in every direction, then $(X,\|\cdot\|)$ is strictly convex [4], i.e., if for any $x, y \in X$ and $\alpha \in[0,1]$, we have $\|z-x\| \leqslant(1-\alpha)\|x-y\|$ and $\|z-y\| \leqslant \alpha\|x-y\|$, then $z=\alpha x+(1-\alpha) y$.

The following technical lemma will be crucial to the proof of the main result of this section.

Lemma 4.2. Assume $(\mathrm{X},\|\cdot\|)$ is uniformly convex in every direction. Let $\mathrm{C}$ be a weakly compact convex nonempty subset of $\mathrm{X}$. Let $\mathcal{F}=\{\mathrm{T}(\mathrm{t})\}_{\mathrm{t} \geqslant 0}$ be a monotone nonexpansive semigroup defined on $\mathrm{C}$. Let $\mathrm{K}$ be a nonempty closed convex subset of $\mathrm{C}$. Fix $\mathrm{x}_{0} \in \mathrm{C}$. Define the function $\varphi: \mathrm{K} \rightarrow[0,+\infty)$ by

$$
\varphi(x)=\limsup _{t \rightarrow \infty}\left\|T(t) x_{0}-x\right\|=\inf _{s \geqslant 0}\left(\sup _{t \geqslant s}\left\|T(t) x_{0}-x\right\|\right) .
$$

Then there exists a unique $z \in \mathrm{K}$ such that $\varphi(z)=\inf _{x \in \mathrm{K}} \varphi(x)$.

Proof. First, let us discuss some of the properties satisfied by $\varphi$.

(a) For any $s \in[0, \infty)$ and $x, y \in K$, we have

$$
\sup _{t \geqslant s}\left\|T(t) x_{0}-x\right\| \leqslant \sup _{t \geqslant s}\left\|T(t) x_{0}-y\right\|+\|x-y\|
$$

which implies $|\varphi(x)-\varphi(y)| \leqslant\|x-y\|$. In other words, $\varphi$ is uniformly continuous. In a similar fashion, we have

$$
\|x-y\| \leqslant \varphi(x)+\varphi(y)
$$

for any $x, y \in K$.

(b) For any $s_{1}, s_{2} \in[0, \infty), x, y \in K$, and $\alpha \in[0,1]$, set $s=\max \left\{s_{1}, s_{2}\right\}$, then we have

$$
\sup _{t \geqslant s}\left\|T(t) x_{0}-(\alpha x+(1-\alpha) y)\right\| \leqslant \alpha \sup _{t \geqslant s_{1}}\left\|T(t) x_{0}-x\right\|+(1-\alpha) \sup _{t \geqslant s_{2}}\left\|T(t) x_{0}-y\right\|,
$$

which implies

$$
\varphi(\alpha x+(1-\alpha) y) \leqslant \alpha \sup _{t \geqslant s_{1}}\left\|T(t) x_{0}-x\right\|+(1-\alpha) \sup _{t \geqslant s_{2}}\left\|T(t) x_{0}-y\right\| .
$$

Since $s_{1}$ and $s_{2}$ are arbitrarily positive numbers, we get

$$
\varphi(\alpha x+(1-\alpha) y) \leqslant \alpha \varphi(x)+(1-\alpha) \varphi(y),
$$

i.e., $\varphi$ is convex.

Set $\varphi_{0}=\inf _{x \in K} \varphi(x)$. For any $n \geqslant 1$, the set $K_{n}=\left\{x \in K ; \varphi(x) \leqslant \varphi_{0}+1 / n\right\}$ is a closed and convex nonempty subset of $K$. Since $C$ is weakly compact and $K$ is closed, we conclude that $K_{\infty}=\bigcap_{n \geqslant 1} K_{n}$ is nonempty. Clearly, for any $z \in K_{\infty}$, we have $\varphi(z)=\varphi_{0}$. Therefore, $\varphi$ attains its minimum. Next, we prove that $K_{\infty}$ is reduced to one point. It follows from (a) above that if $\varphi_{0}=0$, then $K_{\infty}$ is reduced to one point. Assume otherwise that $\varphi_{0}>0$ and $z_{1}, z_{2} \in K_{\infty}$ with $z_{1} \neq z_{2}$. Set $z=\left(z_{1}-z_{2}\right) /\left\|z_{1}-z_{2}\right\|$. Since $(X,\|\cdot\|)$ is uniformly convex in every direction, for any $\varepsilon>0$, there exists $\delta(\varepsilon, z)>0$ such that

$$
\left\|\frac{x+y}{2}\right\| \leqslant 1-\delta(\varepsilon, z)
$$


for any $x, y \in X$ such that

$$
\|x\| \leqslant 1,\|y\| \leqslant 1, \quad x-y=\alpha z, \text { and }\|x-y\| \geqslant \varepsilon .
$$

Let $\eta<\varphi_{0}$. Then by definition of $\varphi$, there exists $s_{0} \geqslant 0$ such that

$$
\sup _{t \geqslant s_{0}}\left\|T(t) x_{0}-z_{\mathfrak{i}}\right\|<\varphi_{0}+\eta
$$

for $i=1,2$. Fix any $t \geqslant s_{0}$. Set

$$
x=\frac{T(t) x_{0}-z_{1}}{\varphi_{0}+\eta} \text { and } y=\frac{T(t) x_{0}-z_{2}}{\varphi_{0}+\eta} .
$$

Then we have $\|x\| \leqslant 1$ and $\|y\| \leqslant 1$. Moreover, we have

$$
y-x=\frac{z_{1}-z_{2}}{\varphi_{0}+\eta} z \text { and }\|y-x\|=\frac{\left\|z_{1}-z_{2}\right\|}{\varphi_{0}+\eta} \geqslant \frac{\left\|z_{1}-z_{2}\right\|}{2 \varphi_{0}}
$$

Using (UCED), we get

$$
\left\|\frac{x+y}{2}\right\| \leqslant 1-\delta\left(\frac{\left\|z_{1}-z_{2}\right\|}{2 \varphi_{0}}, z\right)
$$

which implies

$$
\left\|\mathrm{T}(\mathrm{t}) \mathrm{x}_{0}-\frac{z_{1}+z_{2}}{2}\right\| \leqslant\left(\varphi_{0}+\eta\right)\left(1-\delta\left(\frac{\left\|z_{1}-z_{2}\right\|}{2 \varphi_{0}}, z\right)\right) .
$$

Since $t$ was chosen arbitrarily larger than $s_{0}$, we get

$$
\sup _{t \geqslant s_{0}}\left\|\mathrm{~T}(\mathrm{t}) \mathrm{x}_{0}-\frac{z_{1}+z_{2}}{2}\right\| \leqslant\left(\varphi_{0}+\eta\right)\left(1-\delta\left(\frac{\left\|z_{1}-z_{2}\right\|}{2 \varphi_{0}}, z\right)\right),
$$

which implies

$$
\varphi\left(\frac{z_{1}+z_{2}}{2}\right) \leqslant\left(\varphi_{0}+\eta\right)\left(1-\delta\left(\frac{\left\|z_{1}-z_{2}\right\|}{2 \varphi_{0}}, z\right)\right) .
$$

If we let $\eta \rightarrow 0$, we get

$$
\varphi_{0} \leqslant \varphi\left(\frac{z_{1}+z_{2}}{2}\right) \leqslant \varphi_{0}\left(1-\delta\left(\frac{\left\|z_{1}-z_{2}\right\|}{2 \varphi_{0}}, z\right)\right)<\varphi_{0} .
$$

This contradiction proves that $K_{\infty}$ is reduced to one point. Therefore, $\varphi$ has a unique minimum in $K$ as claimed.

Now we are ready to state the main result of this section. This theorem generalizes Kozlowski's result in [13] from the uniform convex case to the case of $X$ being uniformly convex in every direction. As described at the beginning of this section, such a generalization is non-trivial and of significant importance.

Theorem 4.3. Assume $(\mathrm{X},\|\cdot\|)$ is uniformly convex in every direction. Let $\mathrm{C}$ be a weakly compact convex nonempty subset of $\mathrm{X}$. Let $\mathcal{F}=\{\mathrm{T}(\mathrm{t})\}_{\mathrm{t} \geqslant 0}$ be a monotone nonexpansive semigroup defined on $\mathrm{C}$. Assume there exists $\mathrm{x}_{0} \in \mathrm{C}$ such that $x_{0} \preceq \mathrm{T}(\mathrm{t}) \mathrm{x}_{0}$ (resp. $\mathrm{T}(\mathrm{t}) \mathrm{x}_{0} \preceq \mathrm{x}_{0}$ ) for any $\mathrm{t} \geqslant 0$. Then there exists a common fixed point $z \in \operatorname{Fix}(\mathcal{F})$ such that $x_{0} \preceq z$ (resp. $\left.z \preceq x_{0}\right)$.

Proof. Without loss of generality, we assume $x_{0} \preceq T(t) x_{0}$ for any $t \geqslant 0$. First notice that

$$
K=\bigcap_{t \geqslant 0}\left(\left[T(t) x_{0}, \rightarrow\right) \cap C\right)
$$

is a nonempty closed convex subset of $\mathrm{C}$. Since order intervals are convex and closed, the statement is 
true if we prove that $\mathrm{K}$ is nonempty. For this, we will use the weak compactness of $\mathrm{C}$. It is enough to only prove that $\bigcap_{i \in\{1, \cdots, n\}}\left(\left[T\left(t_{i}\right) x_{0}, \rightarrow\right) \cap C\right) \neq \emptyset$ for any arbitrary numbers $t_{1}, \cdots, t_{n} \in[0,+\infty)$. Set $x=T\left(t_{1}+\cdots+t_{n}\right) x_{0} \in C$. Since the semigroup is monotone and $x_{0} \preceq T(t) x_{0}$ for any $t \geqslant 0$, then $T(s) x_{0} \preceq T(s+t) x_{0}$ for any $s, t \geqslant 0$. This will imply $T\left(t_{i}\right) x_{0} \preceq x$ for any $i=1, \cdots, n$, i.e.,

$$
x \in\left[T\left(t_{i}\right) x_{0}, \rightarrow\right) \cap C, i=1, \cdots, n .
$$

Hence $\bigcap_{i \in\{1, \cdots, n\}}\left(\left[T\left(t_{i}\right) x_{0}, \rightarrow\right) \cap C\right) \neq \emptyset$ holds. Next, we consider the function $\varphi: K \rightarrow[0,+\infty)$ defined by

$$
\varphi(x)=\limsup _{t \rightarrow \infty}\left\|T(t) x_{0}-x\right\|=\inf _{s \geqslant 0}\left(\sup _{t \geqslant s}\left\|T(t) x_{0}-x\right\|\right)
$$

Using Lemma 4.2, there exists a unique $z \in \mathrm{K}$ such that $\varphi(z)=\inf _{\mathrm{x} \in \mathrm{K}} \varphi(\mathrm{x})$. We claim that $z$ is a common fixed point of the semigroup $\mathcal{F}$. Let $x \in K$ and $s, t \geqslant 0$. If $t \geqslant s$, the inequality $T(t-s) x_{0} \preceq x$ implies $T(t) x_{0} \preceq T(s) x$. Otherwise, assume $t<s$. Set $\varepsilon=s-t$. Since $x_{0} \preceq x$, we get $x_{0} \preceq T(\varepsilon) x_{0} \preceq T(\varepsilon) x$, which implies $T(t) x_{0} \preceq T(t) \circ T(\varepsilon) x=T(t+\varepsilon) x=T(s) x$. Therefore, we have $T(t) x_{0} \preceq T(s) x$ for any $t, s \geqslant 0$, which implies $T(s) x \in K$ for any $s \geqslant 0$. In other words, $K$ is invariant by the semigroup $\mathcal{F}$. Fix $s, \eta \geqslant 0$ and let $t \geqslant s+\eta$. Since $T(t-s) x_{0} \preceq z$, we get

$$
\left\|\mathrm{T}(\mathrm{s}) \circ \mathrm{T}(\mathrm{t}-\mathrm{s}) \mathrm{x}_{0}-\mathrm{T}(\mathrm{s}) z\right\| \leqslant\left\|\mathrm{T}(\mathrm{t}-\mathrm{s}) \mathrm{x}_{0}-z\right\| \leqslant \sup _{\overline{\mathrm{t}} \geqslant \eta}\left\|\mathrm{T}(\overline{\mathrm{t}}) \mathrm{x}_{0}-z\right\|,
$$

which implies $\left\|T(t) x_{0}-T(s) z\right\| \leqslant \sup _{\bar{t} \geqslant \eta}\left\|T(\bar{t}) x_{0}-z\right\|$ for any $t \geqslant s+\eta$. Hence

$$
\varphi(T(s) z) \leqslant \sup _{t \geqslant s+\eta}\left\|T(t) x_{0}-T(s) z\right\| \leqslant \sup _{\bar{t} \geqslant \eta}\left\|T(\bar{t}) x_{0}-z\right\|
$$

Since $\eta$ was taken arbitrarily positive, we get $\varphi(T(s) z) \leqslant \varphi(z)$. Lemma 4.2 will force $T(s) z=z$ by the uniqueness of the minimum point in K. Since $s$ was taken arbitrarily positive, we conclude that $z \in \operatorname{Fix}(\mathcal{F})$ as claimed. Since $x_{0} \preceq z$, the proof of Theorem 4.3 is complete.

Remark 4.4. In fact, the common fixed point set $\operatorname{Fix}(\mathcal{F})$ has a nice geometrical property. Indeed, let $z_{1}, z_{2} \in$ $\operatorname{Fix}(\mathcal{F})$ be comparable. Fix $\alpha \in[0,1]$. Let us prove that $z=\alpha z_{1}+(1-\alpha) z_{2} \in \operatorname{Fix}(\mathcal{F})$. Assume $z_{1} \preceq z_{2}$. Then we have $z_{1} \preceq z \preceq z_{2}$. Since $\mathcal{F}$ is monotone nonexpansive, we get

$$
\left\|\mathrm{T}(\mathrm{t}) z-z_{1}\right\|=\left\|\mathrm{T}(\mathrm{t}) z-\mathrm{T}(\mathrm{t}) z_{1}\right\| \leqslant\left\|z-z_{1}\right\|=(1-\alpha)\left\|z_{1}-z_{2}\right\|,
$$

and similarly $\left\|\mathrm{T}(\mathrm{t}) z-z_{2}\right\| \leqslant \alpha\left\|z_{1}-z_{2}\right\|$ for any $t \geqslant 0$. Since $(X,\|\|$.$) is uniformly convex in every direction,$ then $(X,\|\cdot\|)$ is strictly convex. Therefore, we must have $T(t) z=\alpha z_{1}+(1-\alpha) z_{2}=z$, i.e., $z \in \operatorname{Fix}(T(t))$ for any $t \geqslant 0$ as claimed. This result was known for a single map [6, 7].

\section{Acknowledgment}

The authors would like to extend their sincere appreciation to the Deanship of Scientific Research at King Saud University for funding the Research group No. (RG-1435-079).

\section{References}

[1] M. R. Alfuraidan, M. A. Khamsi, Fibonacci-Mann iteration for monotone asymptotically nonexpansive mappings, Bull. Aust. Math. Soc., 96 (2017), 307-316. 1

[2] M. Bachar, M. A. Khamsi, On common approximate fixed points of monotone nonexpansive semigroups in Banach spaces, Fixed Point Theory Appl., 2015 (2015), 11 pages. 1 
[3] M. Bachar, M. A. Khamsi, Recent contributions to fixed point theory of monotone mappings, J. Fixed Point Theory Appl., 19 (2017), 1953-1976. 1

[4] B. Beauzamy, Introduction to Banach spaces and their geometry, North-Holland Mathematics Studies, Notas de Matemática [Mathematical Notes], North-Holland Publishing Co., Amsterdam-New York, (1982). 4

[5] L. P. Belluce, W. A. Kirk, Nonexpansive mappings and fixed-points in Banach spaces, Illinois J. Math., 11 (1967), 474-479. 3

[6] F. E. Browder, Nonexpansive nonlinear operators in a Banach space, Proc. Nat. Acad. Sci. U.S.A., 54 (1965), 1041-1044. 4.4

[7] R. E. Bruck, Properties of fixed-point sets of nonexpansive mappings in Banach spaces, Trans. Amer. Math. Soc., 179 (1973), 251-262. 3, 4.4

[8] A. L. Garkavi, The best possible net and the best possible cross-section of a set in a normed space, Amer. Math. Soc. Transl. Ser. II, 39 (1964), 111-132. 4.1, 4

[9] K. Goebel, W. A. Kirk, Topics in metric fixed point theory, Cambridge Studies in Advanced Mathematics, Cambridge University Press, Cambridge, (1990). 1

[10] D. Göhde, Zum Prinzip der kontraktiven Abbildung, (German) Math. Nachr., 30 (1965), 251-258. 3

[11] M. A. Khamsi, W. A. Kirk, An introduction to metric spaces and fixed point theory, Pure and Applied Mathematics (New York), Wiley-Interscience, New York, (2001). 1

[12] W. A. Kirk, A fixed point theorem for mappings which do not increase distances, Amer. Math. Monthly, 72 (1965), 1004-1006. 3

[13] W. M. Kozlowski, Monotone Lipschitzian semigroups in Banach spaces, J. Aust. Math. Soc., (in press). 1, 3, 4

[14] T. C. Lim, A fixed point theorem for families on nonexpansive mappings, Pacific J. Math., 53 (1974), 487-493. 3

[15] Z. Opial, Weak convergence of the sequence of successive approximations for nonexpansive mappings, Bull. Amer. Math. Soc., 73 (1967), 591-597. 3

[16] N. H. Pavel, Nonlinear evolution operators and semigroups, Applications to partial differential equations, Lecture Notes in Mathematics, Springer-Verlag, Berlin, (1987). 1

[17] A. Pazy, Semigroups of linear operators and applications to partial differential equations, Applied Mathematical Sciences, Springer-Verlag, New York, (1983). 1

[18] A. C. M. Ran, M. C. B. Reurings, A fixed point theorem in partially ordered sets and some applications to matrix equations, Proc. Amer. Math. Soc., 132 (2004), 1435-1443. 1

[19] F. Vetro, On approximating curves associated with nonexpansive mappings, Carpathian J. Math., 27 (2011), 142-147. 3

[20] F. Vetro, Fixed point results for nonexpansive mappings on metric spaces, Filomat, 29 (2015), 2011-2020. 3

[21] V. Zizler, On some rotundity and smoothness properties of Banach spaces, Dissertationes Math. Rozprawy Mat., 87 (1971), 33 pages. $4.1,4$ 